\title{
STATIC AEROELASTIC ANAL YSIS OF TRANSONIC WIND TUNNEL MODELS USING FINITE ELEMENT METHODS
}

\author{
John R. Hooker* \\ McDonnell Douglas Corporation \\ Long Beach, CA \\ Alpheus W. Burner ${ }^{\dagger}$ \\ NASA Langley Research Center \\ Hampton, VA \\ Robert Valla ${ }^{\ddagger}$ \\ McDonnell Douglas Corporation \\ Long Beach, CA
}

\begin{abstract}
A computational method for accurately predicting the static aeroelastic deformations of typical transonic transport wind tunnel models is described. The method utilizes a finite element method (FEM) for predicting the deformations. Extensive calibration/validation of this method was carried out using a novel wind-off wind tunnel model static loading experiment and wind-on optical wing twist measurements obtained during a recent wind tunnel test in the National Transonic Facility (NTF) at NASA LaRC. Further validations were carried out using a Navier-Stokes computational fluid dynamics (CFD) flow solver to calculate wing pressure distributions about several aeroelastically deformed wings and comparing these predictions with NTF experimental data. Results from this aeroelastic deformation method are in good overall agreement with experimentally measured values. Including the predicted deformations significantly improves the correlation between CFD predicted and experimentally measured wing surface pressures.
\end{abstract}

\section{INTRODUCTION}

The accurate prediction of wind tunnel model static aeroelastic deformations is becoming increasingly important for transonic testing of transport configurations. Wind tunnel model static aeroelastic deformations are defined as steady state deformations due to aerodynamic forces acting on elastic wind tunnel models. In order to illustrate the importance of accurately predicting these model deformations, the procedure used to incorporate them into the wind tunnel model design is briefly described. First, the $1-\mathrm{g}$ wing that is to be tested is defined, usually using a CFD based design tool. Then, a FEM is utilized to predict the deformations (twist and bending) that the model will undergo under a given loading condition (typically mid-cruise). And finally, these predicted deformations are "subtracted" from the $1-g$ wing in order to define the model jig wing, which will deform into the $1-\mathrm{g}$ shape at the given loading condition. Therefore, it is essential to accurately predict the model static aeroelastic deformations in the wind tunnel in order to duplicate the desired CFD configuration. Also, deformations must often be taken into account when comparing CFD predictions to experimental measurements at off-design conditions. For example, the comparison of CFD predictions to wind tunnel measurements at buffet conditions requires the definition of a new CFD wing which accounts for the increased model deformations associated with this higher loading condition. Since increased reliance is being placed on high Reynolds number (Re) testing for configuration development and for CFD validation,' the accurate prediction of static aeroelastic deformations has become increasingly important, because the deformations increase significantly at the higher dynamic pressures associated with high Re testing.

The objective of this paper is to describe the application of a FEM for the accurate prediction of static aeroelastic deformations of a transonic low wing transport wind tunnel model and to present several results from an extensive calibration effort. Several researchers have utilized FEMs in the past to predict the static

\footnotetext{
- Senior Engineer, Advanced Systems and Technology, Member AIAA

${ }^{+}$Physicist, Experimental Testing Technology Division, Member AIAA

\$ Senior Engineer, Advanced Systems and Technology, Member AIAA

Copyright 1997 by the American Institute of Aeronautics and Astronautics, Inc. All rights reserved.
} 
aeroelastic deformations of either production aircraft or wind tunnel models. ${ }^{2,3}$ However, McDonnell Douglas Corporation (MDC) has recently developed a unique database for calibrating/validating these types of computational methods. This database consists of wing twist and bending data obtained from a novel wind-off model static loading experiment and wind-on optical wing twist measurements recorded during a recent NTF wind tunnel test and was utilized extensively to calibrate/validate the FEM described herein.

\section{METHODS}

The computational and experimental methods utilized to determine the aeroelastic deformations of an advanced wing (transonic low wing transport) NTF wind tunnel model is described. This model was recently tested at both low and high $\mathrm{Re}$ in the NTF with and without nacelles/pylons installed. Both the left and right hand wings were pressure instrumented and consequently contained extensive spanwise and chordwise pressure tube troughing. The definition of the spanwise troughing was provided by the model builder, but the chordwise finger troughing was not available because it was hand-worked and consequently did not exist in the $\mathrm{CAD}$ surface definition used to machine the wing.

\section{Computational}

The solver used in this effort to predict aeroelastic deformations is the Aeroelastic Design Optimization

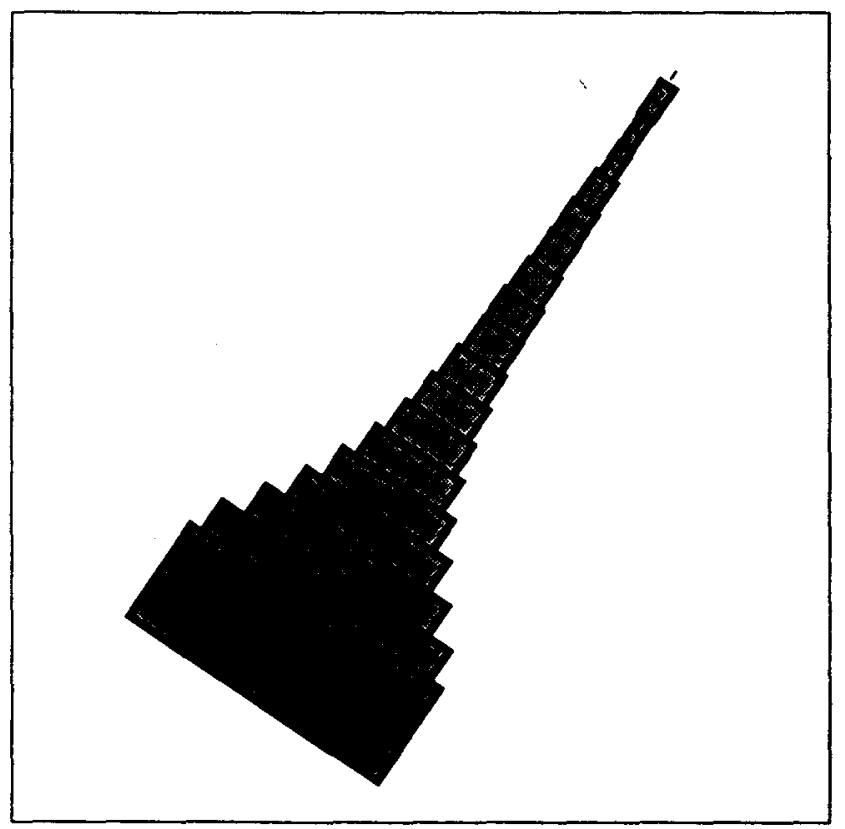

Figure 1: Depiction of the 1-D FEM grid used to calculate the 1-D ADOP deformations.
Program (ADOP), which is a NASTRAN-like, MDCdeveloped FEM. For this effort ADOP was run in both the 1-D beam and 3-D solid modes (both of which modeled the spanwise pressure tube troughing of the model). One of the advantages of the simple 1-D beam analysis method is that the grid generation is simple, as can be seen in Figure 1. Each of the beam elements was sized to approximate the sectional wing stiffness determined using CAD. Another advantage of this $1-D$ method is its inherent fast turn around time, which allows the user to evaluate several loading conditions in a relatively short period of time. However, since it is a simplistic model, its accuracy needs to be thoroughly evaluated. The main advantage of the more advanced 3D solid analysis method is that its accuracy is expected to be better. However, there are several disadvantages associated with this method. First, the grid generation is very complex. For example, the generation of the 3D hexahedral volume grid depicted in Figure 2 (which incorporates all the available model cut-outs and troughing) required approximately one month for an experienced ADOP user. Also, significant user time is required to modify the loadings in order to evaluate different flight conditions. And finally, it requires a very detailed model definition up front. These disadvantages tend to make this method undesirable for defining wind tunnol model jig wings since it would lengthen the model definition effort considerably, and consequently, the aerodynamic design cycle time.

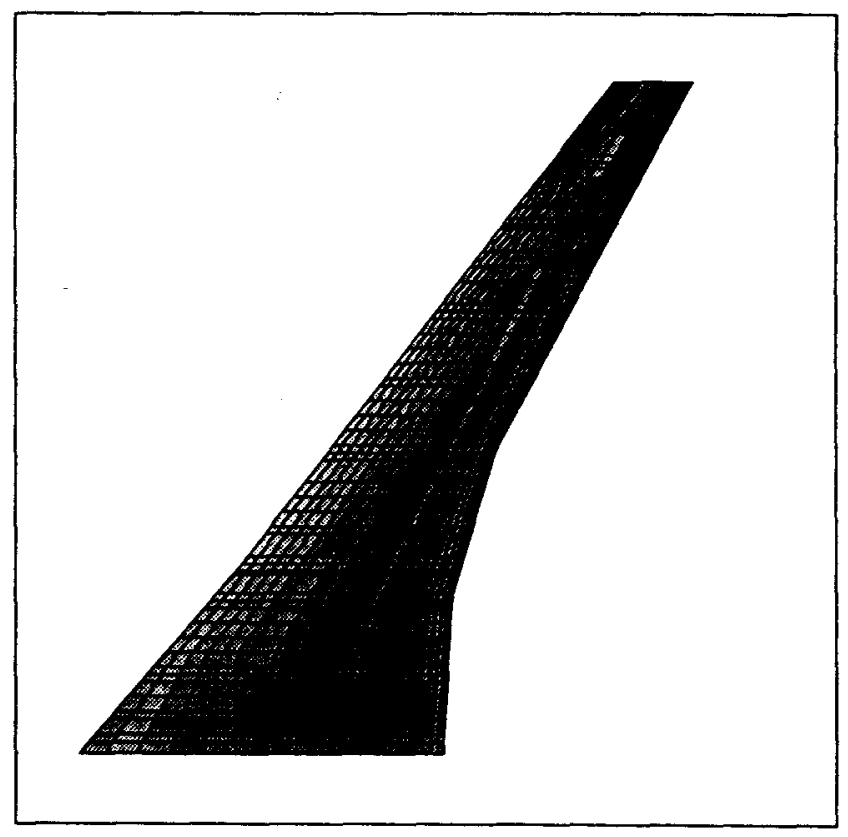

Figure 2: Depiction of the 3-D FEM grid used to calculate the 3-D ADOP deformations. 


\section{Experimental}

The experimental wing twist measurements were obtained in the NTF using an automated optical measurement technique." The basic instrumentation consists of a single charge-coupled device (CCD) video camera and a frame grabber interfaced to a personal computer. The technique is based upon a single view photogrammetric determination of two dimensional coordinates of wing targets with a fixed (and known) third dimensional coordinate, namely the spanwise location. The optical measurement technique has been used successfully for a variety of tests at not only the NTF, but also the Langley Transonic Dynamics Tunnel, the Langley Unitary Plan Wind Tunnel, and the NASA Ames 12 Foot Pressure Wind Tunnel. Additional examples of measurements made at the NTF can be found in Reference 5 . The uncertainty of the optical wing twist measurements can be expressed in terms of precision, or repeatability, and bias, or systematic, errors. Bias errors are, in general, very difficult to determine under flow conditions, whereas the repeatability can be evaluated. The short term repeatability is influenced by factors such as the resolution of the targets and the sampling rate of the optical measurements (which is especially important at buffet conditions where the model dynamics are significant) and can be assessed by evaluating the runto-run repeatability of the optical wing twist measurements. The long term repeatability is influenced by factors such as the accuracy of the determination of the camera position and viewing angles and the wind-off polars, which are used as a reference for angle calibrations based upon the onboard accelerometer. The long term repeatability is improved by using wind-off polars which bracket each set of runs and by minimizing the time between the wind-on and wind-off measurements. In a cryogenic environment, it is especially important to avoid any significant time lags between the wind-off and wind-on measurements, since during these delays, the nitrogen injection is often minimized for economic reasons and the resulting thermal gradients can lead to tunnel movement which will introduce errors. The long term repeatability can be assessed by evaluating the test-to-test repeatability of the optical wing twist measurements.

For the advanced wing NTF model, high contrast white targets were placed on a flat black background strip at the $95 \%$ span station, as depicted in Figure 3. The targets were placed at a single station, rather that at several wing stations, in order to reduce the camera's field of view, thus increasing the optical resolution of the targets and thereby reducing precision errors. Consequently, wing twist measurements were only made at the $95 \%$ span station. The mean surface roughness of the polished paint targets and background strip was measured with a profilometer and was found

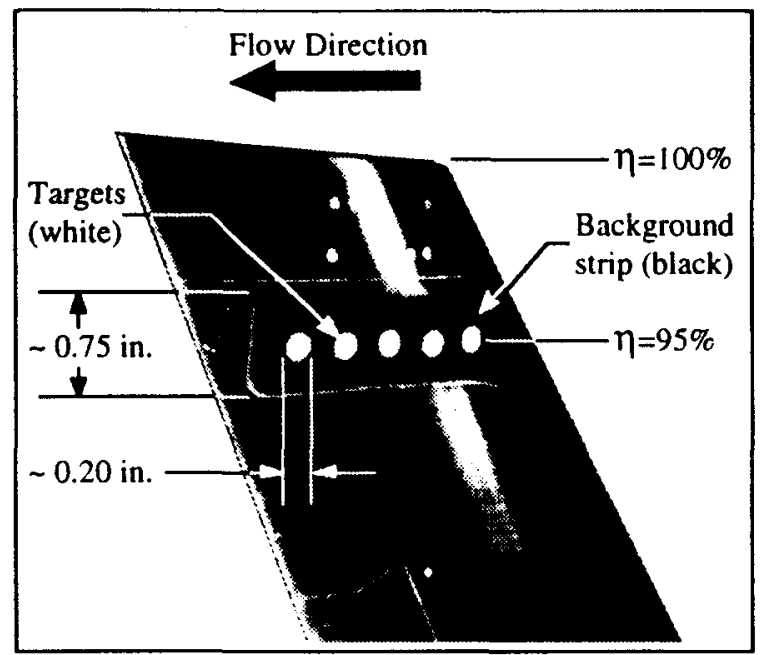

Figure 3: Background strip and targets applied to the advanced wing NTF model.

to be approximately 10 microinch $\mathrm{rms}$, which is within the surface finish requirements of typical NTF transonic models. The total paint thickness was approximately 0.002 inches and was feathered along the border to provide a gradual transition to the 0.002 inch thickness and eliminate an abrupt step.

\section{RESULTS AND DISCUSSION}

\section{Wind-Off Static Loading Experiment}

A static loading experiment was devised to calibrate/validate both the NTF optical measurement system and the FEM analysis methods. When developing this experiment it was recognized that it is not necessary to simulate the actual wind-on loadings which the model undergoes during testing, but simply to apply consistent loadings on the model and in the FEM analysis, loadings which result in measurable model deformations. . This experiment, which is depicted schematically in Figure 4, consisted of a single point load applied to the advanced wing wind tunnel model at the $95 \%$ span station and the $\mathrm{x} / \mathrm{c}$ location of the elastic axis while the model was mounted in the test section of the NTF so that optical wing twist measurements could be recorded. Mechanical twist measurements were recorded using an accelerometer with an accuracy of $\pm 0.01^{\circ}$ attached to the wing tip tang. Also, as depicted in Figure 4, a fairly large load block (1" in diameter, approximately one third of the local wing chord) was inserted between the point load and the wing lower surface to prevent any model damage. The impact of this load block is that the load applied to the wing is no longer strictly a point load, but is in reality a distributed load over the 1" diameter block. 


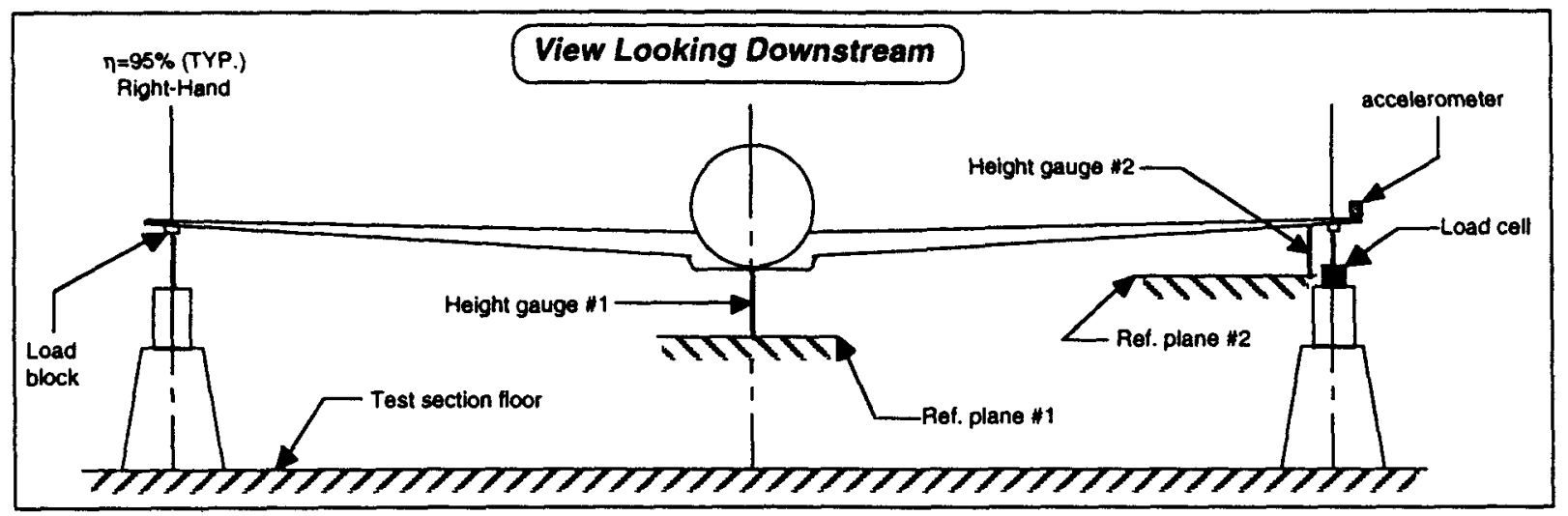

Figure 4: Schematic Depiction of the wind-off static loading experiment.

Results from this experiment are depicted in Figure 5 with wing twist data from the mechanical measurements, the NTF optical measurement system, and the 3-D and 1-D ADOP FEM analyses. From this figure, which depicts the wing twist at the $95 \%$ span station as a function of the applied point load, it can be seen that the NTF optical wing twist measurements correlate reasonably well with the mechanical measurements, within approximately $5 \%$ throughout the loading range tested. Close examination of Figure 5 reveals that there are actually two sets of NTF optical measurements: a baseline and a repeat run. The repeat run was performed immediately upon completion of the baseline run and the good correlation between the two runs indicates that short term repeatability errors are not significant. However, bias errors (possibly due to uncertainties in the determination of the camera position and viewing angles) may account for the 5\% discrepancy. It can also be seen in Figure 5 that there is fair correlation between the 1-D and 3-D ADOP results and the mechanical measurements. Twist results from the 3-D ADOP analysis method were within approximately $15 \%$ of the mechanical measurements, while those from the 1-D ADOP analysis method were within approximately $20 \%$. It is currently believed, however, that approximately $5 \%$ of this discrepancy between the ADOP predicted and mechanical wing twist measurements is caused by how the point load was applied to the wind tunnel model through a relatively large load block. As noted previously, the use of a load block attached to the wing results in a distributed load being applied to the wing, instead of a point load. This is not a problem when there are no deformations (or the deformations are very small) because the distributed load can be resolved as a point load applied at the center of the block. However, as the wing deforms (bends up and washes out) the center of pressure of this distributed load will no longer be at the center of the disk, but will move aft and outboard. This $5 \%$ estimate for the discrepancy caused by the load block was arrived at after evaluating, with the FEMs, the impact of a movement in the point of application of the load. It is important to note that the point load movement does not impact the correlation between the mechanical and optical measurements since these measurements were both made at the same time under consistent loading conditions. It is also currently believed that a significant portion of the remaining discrepancy between the ADOP predicted and mechanical wing twist measurements is due to the inability to model the chordwise pressure tube troughings, as discussed previously.

\section{Wind-On Wing Twist Predictions and Measurements}

Wind-on wing twist data from the 1-D and 3-D ADOP FEM analyses is depicted in Figure 6 along with NTF optical measurements, where the wing twist at the $95 \%$ span station has been plotted as a function of the lift coefficient. The ADOP results were generated using wind tunnel span loadings obtained by integrating wing pressures to determine the sectional loading characteristics. This data was interpolated to discreet span stations, integrated to determine point loads, and applied to the FEM model. This procedure was followed to ensure that the loads applied in the FEM were consistent with those seen by the model. Once again, the experimental wing twist measurements were obtained in the NTF using the optical measurement system. The optical data depicted in Figure 6 represents all the low $\operatorname{Re}$ wing twist measurements obtained at Mach 0.85 and shows that the day-to-day repeatability of the NTF optical measurement system at low $\operatorname{Re}$ is within $0.1^{\circ}$. The nacelle/pylon installed data (WBNP) was included because previous experience has indicated that the installation of the nacelle and pylon has only a minimal impact on the wing aeroelastics. From this figure it can also be seen that both the 1-D and 3-D ADOP predictions agree well with the optical measurements, with the 3-D ADOP results having slightly better correlation. Once again, these FEM predictions would be improved if the chordwise 


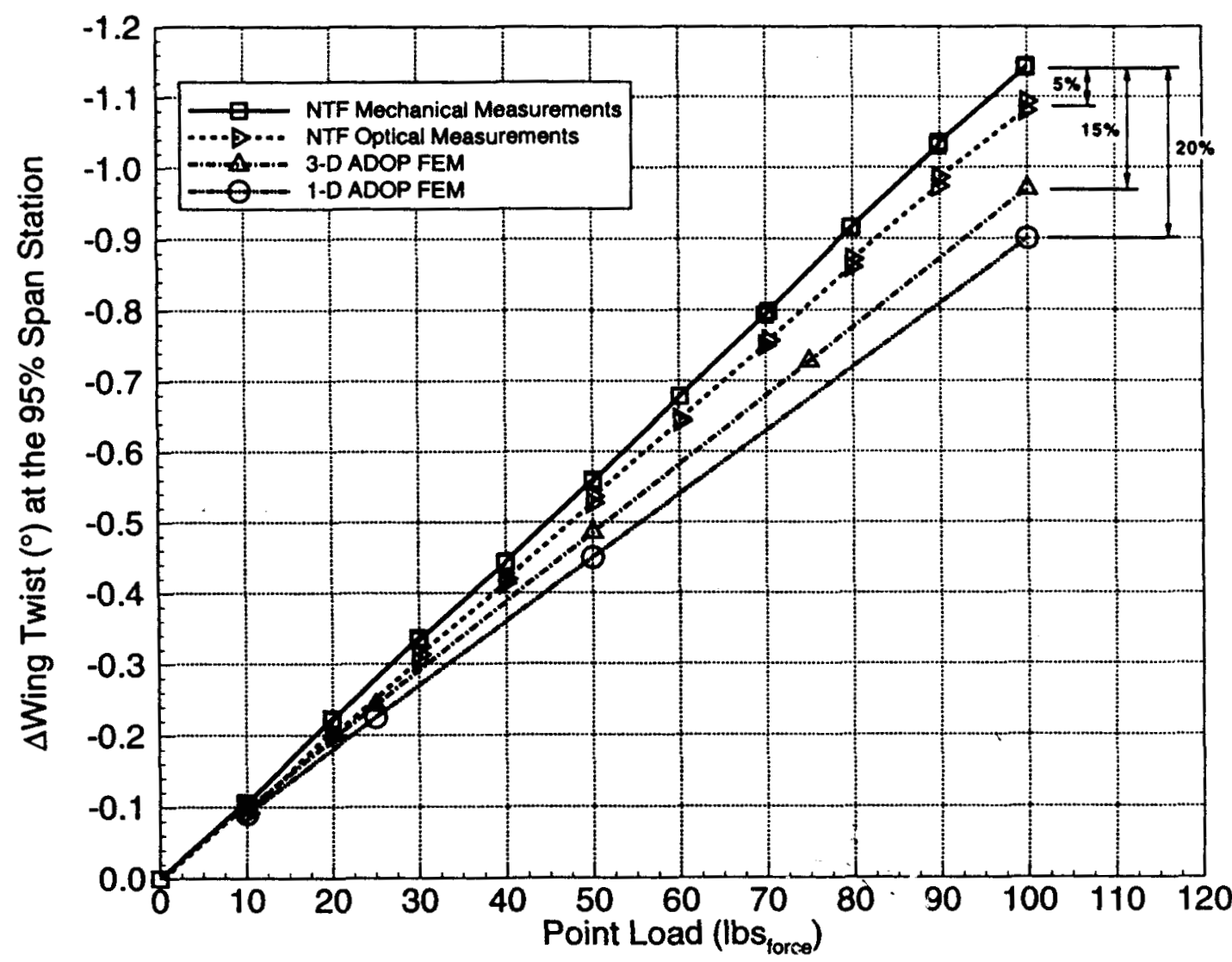

Figure 5: Wing twist results at the $95 \%$ span station from the wind-off static loading experiment.

pressure tube troughings could be modeled since accounting for them would reduce the stiffiness of the wing, thereby increasing the wing deformations.

To quantify the impact of the predicted aeroelastic deformations on CFD solutions, the deformations at the mid-cruise condition were further investigated. Depicted in Figure 7 is the 1-D and 3-D ADOP predicted wing twist and bending along the span of the advanced wing NTF model at low Re mid-cruise conditions. From this figure it can be seen that the wing twist correlation along the entire span is within approximately $0.05^{\circ}$. To quantify the impact of these differences on CFD predictions, CFL3D (a structured grid Navier-Stokes flow solver described in Reference 6) solutions were generated at mid-cruise conditions on the model jig wing/body (WB) configuration (i.e. assuming no aeroelastic deformations) and on the WB configurations defined with the 1-D and 3-D ADOP predicted mid-cruise twist and bending distributions. Results from these analyses are depicted in Figure 8 along with NTF measured wing surface pressure distributions obtained at mid-cruise conditions. From this figure, it can be seen that there is no discernible difference between the two CFD solutions generated on the configurations defined with the 1-D and 3-D ADOP predicted twist and bending distributions and that the correlation with the NTF data is overall very good (especially on the inboard portion of the wing). The only region where there are noticeable differences with the measured data is in the outboard most regions, where the CFD overpredicts the roof top level. This is consistent with an underprediction of the incremental wing twist, since an increase in the wing twist would reduce the angle of attack of the section thereby reducing the roof top level and improving the correlation. This indicates that a slight discrepancy in wing twist may still exist near the wing tip (possibly caused by the inability to model the chordwise pressure tube troughings). These results indicate that a simple 1-D beam FEM analysis is sufficient to define wind tunnel model jig wings. From this figure it can also be seen that there are significant differences between the CFD predicted wing surface pressure distributions generated using geometries which include the predicted aeroelastic deformations and the CFD predicted distribution generated on the model jig wing (which does not 


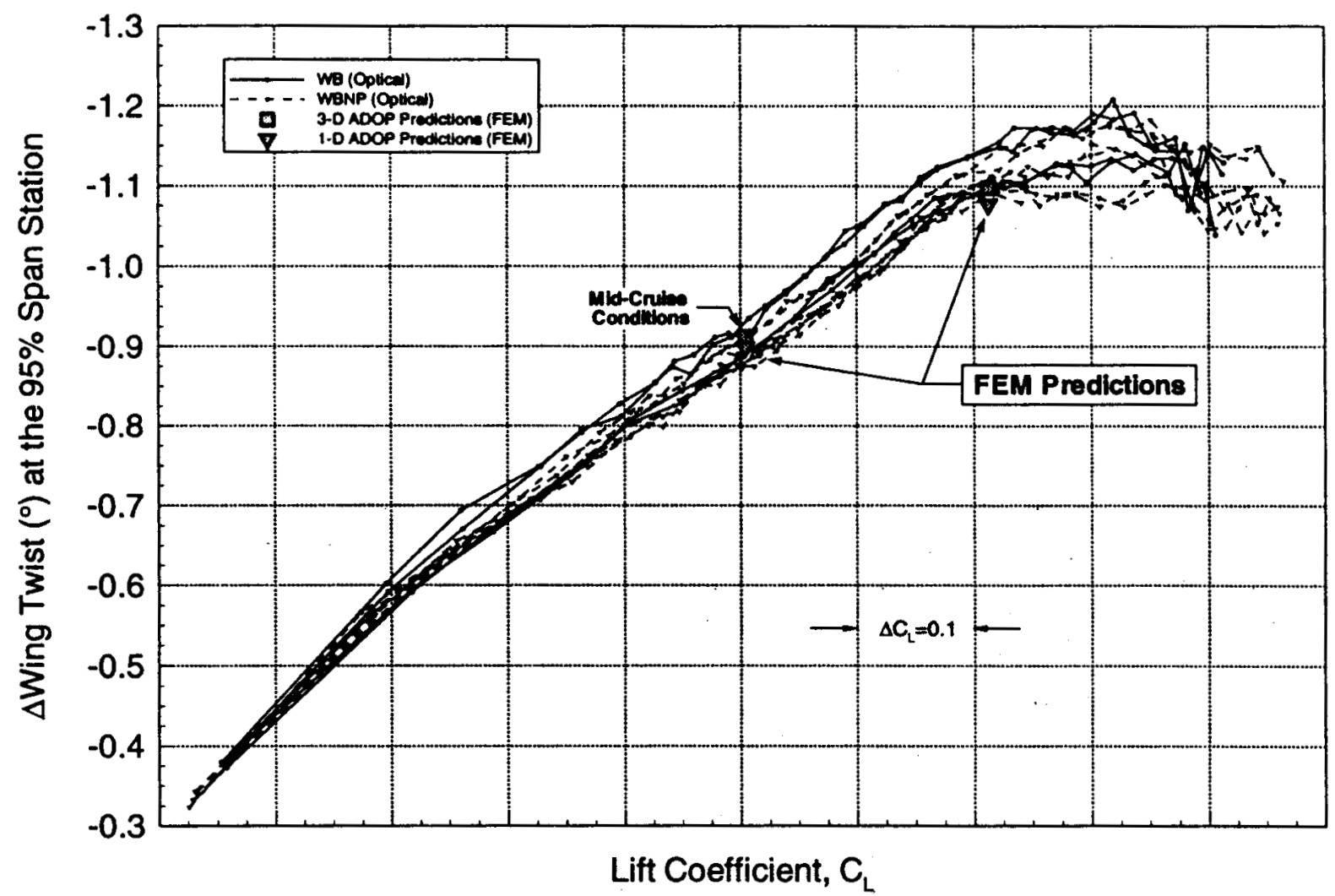

Figure 6: Comparison of wind-on FEM predicted and NTF optically measured wing twist at the $95 \%$ span station (low Re).

include any aeroelastic deformations). This indicates that, even at low $R e$, it is extremely important to include the aeroelastic deformations of the wind tunnel model. Due to the higher dynamic pressures inherent to high $\operatorname{Re}$ testing, it is expected that the aeroelastic effects on the wing flow field would be even greater at such conditions.

\section{CONCLUSIONS}

A FEM for accurately predicting the static aeroelastic deformations of typical transonic low wing transport wind tunnel models has been described. This method was extensively calibrated/validated using a novel wind -off static loading experiment and wind-on optical wing twist measurements obtained during a recent NTF wind tunnel test. Additional validation was carried out using a Navier-Stokes CFD flow solver to calculate solutions about several aeroelastically deformed wings and comparisons were made with NTF experimental data. Results from this aeroelastic deformation method were in good overall agreement with experimentally measured values and it was concluded that a 1-D FEM analysis is sufficient for the definition of wind tunnel model jig wings. However, it is believed that the absence of the chordwise troughing in the FEM model adversely impacted the predicted deformations, highlighting the need for a complete definition of all of the wind tunnel model cut-outs when performing aeroelastic analyses. Including the predicted deformations significantly improved the correlation between CFD predicted and wind tunnel measured wing surface pressures, indicating the importance of modeling these deformations.

\section{FUTURE WORK}

Efforts are currently underway to further expand the FEM calibration/validation database. Additional windon optical wing twist measurements are being obtained from three NTF wind tunnel tests of different transonic configurations. Also, a more detailed wind-off model static loading experiment was recently completed which will provide additional calibration data for both the FEMs and the NTF optical measurement system.

\section{ACKNOWLEDGMENTS}

The authors would like to thank George Tzong and Tom Wu, McDonnell Douglas Corporation, for providing guidance in performing the FEM analyses. The computational grids used to obtain the advanced wing CFD solutions were generated by Yoram Yadlin, McDonnell Douglas Corporation. The advanced wing CFD solutions were generated using the Numerical 
Aerodynamics Simulation (NAS) facility at NASA Ames Research Center. Also, the authors would like to thank the personnel at the NASA LaRC NTF for their assistance in obtaining the wind-off/on model deformation measurements.

\section{REFERENCES}

[1] Lynch, F. T., "Experimental Necessities for Subsonic Transport Configuration Development," AlAA 92-0158, January 1992.

[2] Guruswamy, G., "Coupled Finite-Difference/FiniteElement Approach for Wing-Body Aeroelasticity," AIAA-92-4680, September 1992.

[3] Tzong, T., Chen, H. H., Chang, K. C., Wu, T., Cebeci, T., "A General Interface Method for Aeroelastic Analysis of Aircraft," MDC Report No. MDC 96K7062, February 1996.

[4] Burner, A. W. and Martinson, S.D., "Automated Wing Twist and Bending Measurements Under Aerodynamic Load," AIAA-96-2253, June 1996.

[5] Burner, A. W., Wahls, R. A., Goad, W. K., "Wing Twist Measurements at the National Transonic Facility," NASA TM 110229, February 1996.

[6] Van Leer, B., Thomas, J. L., Roe, P. L., and Newsome, R. W., "A Comparison of Numerical Flux Formulas for the Euler and Navier-Stokes Equations," AIAA-87-1104, June 1987. 


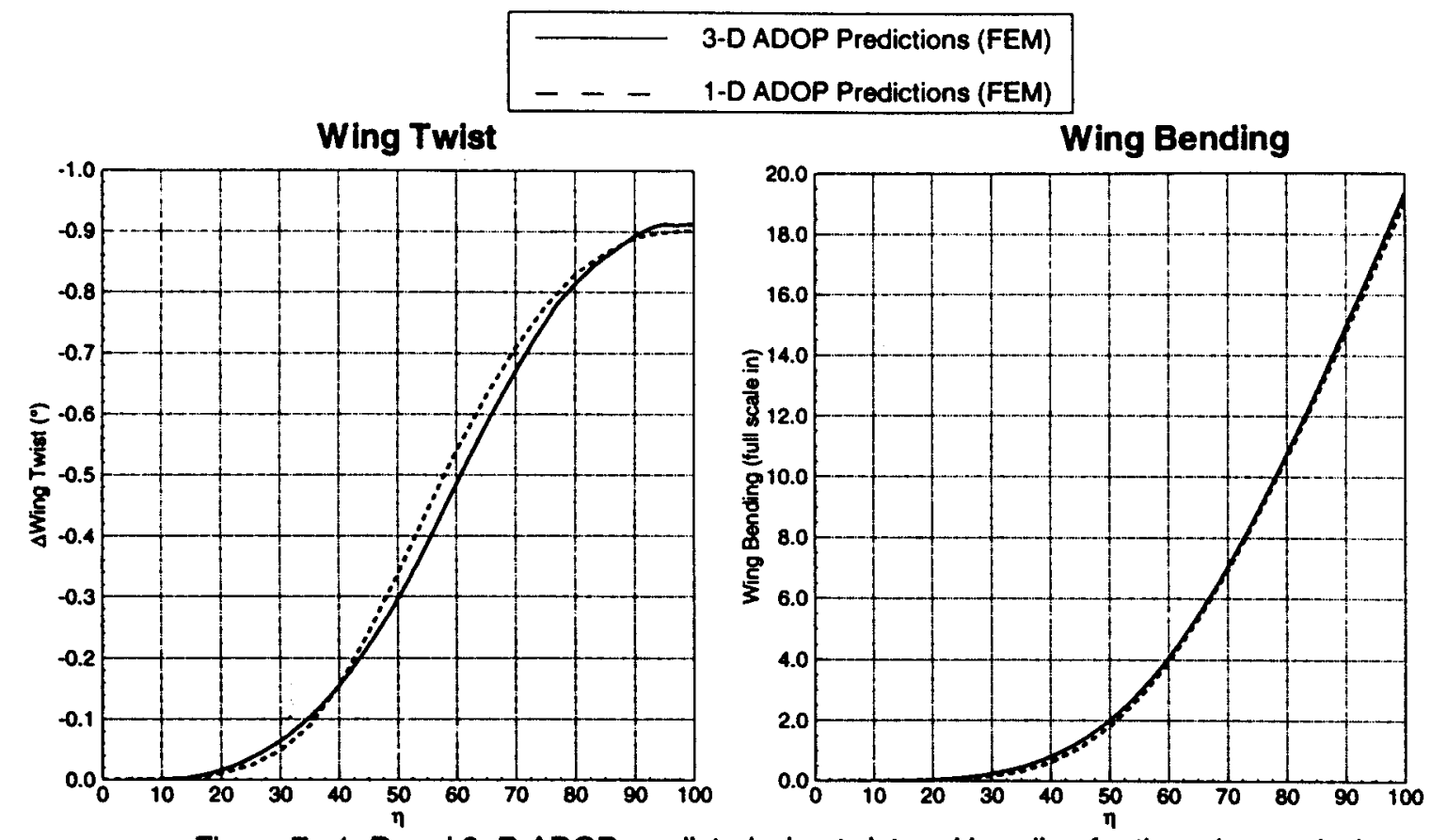

Figure 7: $1-D$ and 3-D ADOP predicted wing twist and bending for the advanced wing NTF model at mid-cruise conditions ( $M=0.85$ and $R e=4.3$ million).
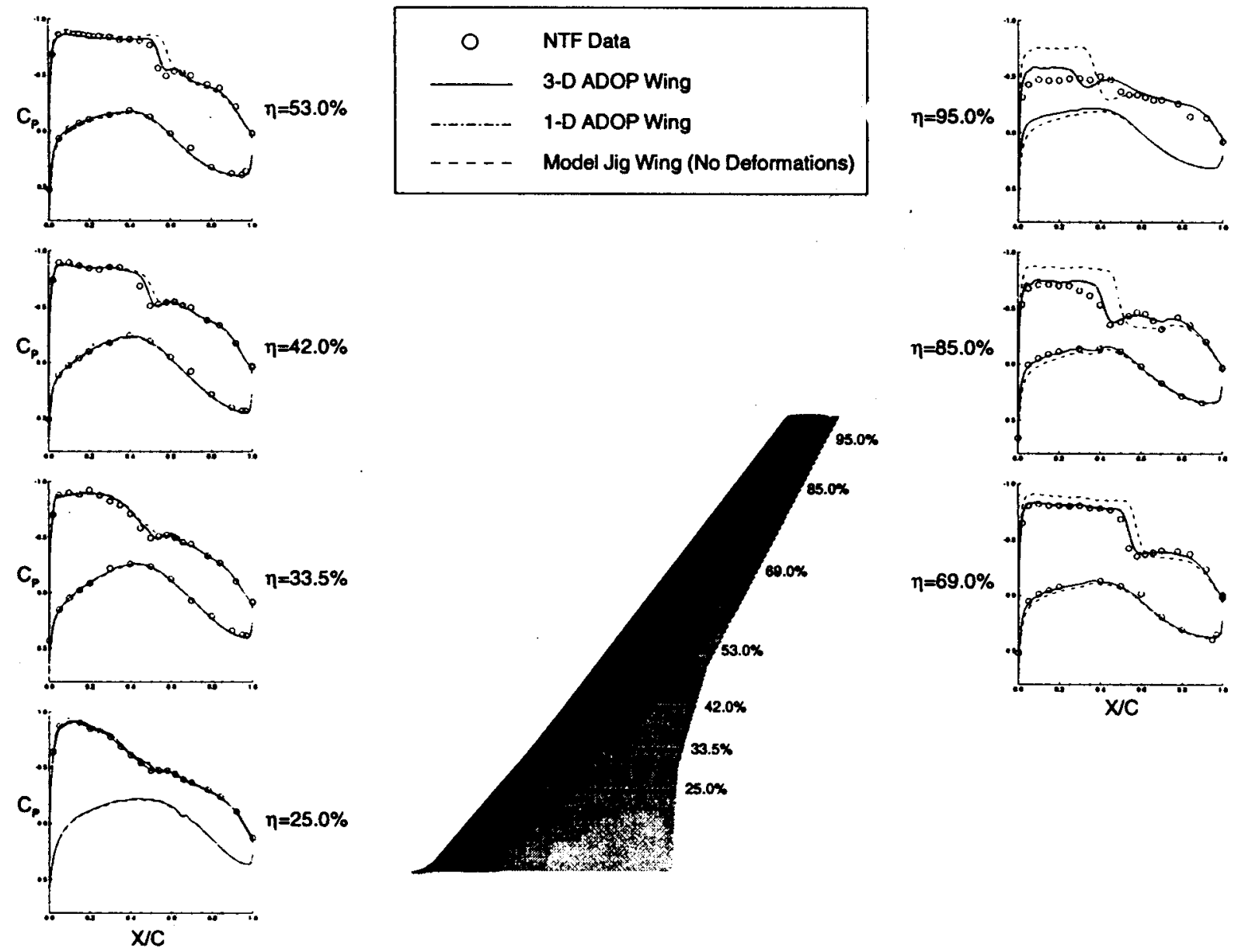

Figure 8: CFL3D predicted wing surface pressure distributions generated on the advanced wing NTF model at mid-cruise conditions ( $M=0.85$ and $R e=4.3$ million) using the model jig wing and aeroelastically deformed wings. 